\title{
The role of the inflammasome in cellular responses to toxins and bacterial effectors
}

\author{
Barbara Freche • Núria Reig • F. Gisou van der Goot
}

Received: 25 May 2007 / Accepted: 6 July 2007 / Published online: 6 September 2007

(C) Springer-Verlag 2007

\begin{abstract}
Invading pathogens are recognized by mammalian cells through dedicated receptors found either at the cell surface or in the cytoplasm. These receptors, like the transmembrane Toll-like Receptors (TLR) or the cytosolic Nod-like Receptors (NLR), initiate innate immunity after recognition of molecular patterns found in bacteria or viruses, such as LPS, flagellin, or double-stranded RNA. Recognition of molecules produced only by a specific pathogen, such as a viral envelop protein or a bacterial adhesin does not appear to occur. Bacterial protein toxins, however, might compose an intermediate class. Considering the diversity of toxins in terms of structure, it is unlikely that cells respond to them via specific molecular recognition. It rather appears that different classes of toxins trigger cellular changes that are sensed by the cells as danger signals, such as changes in cellular ion composition after membrane perforation by pore-forming toxins or type III secretion systems. The signaling pathways triggered through toxin-induced cell alterations will likely play a role in modulating host responses to virulent bacteria. We will here describe the few studied cases in which detection of the toxin by the host cell was addressed. The review will include not only toxins but also bacteria effectors secreted by the bacterium in to the host cell cytoplasm.
\end{abstract}

Keywords Toxins · Inflammasome · Pore-forming ·

Caspase-1 $\cdot$ Effector $\cdot$ Type III secretion

B. Freche $\cdot$ N. Reig $\cdot$ F. G. van $\operatorname{der} \operatorname{Goot}(\bowtie)$

Global Health Institute,

Ecole Polytechnique Fédérale de Lausanne,

Station 15,

1015 Lausanne, Switzerland

e-mail: gisou.vandergoot@epfl.ch
During infection, pathogenic microbes actively modify the behavior of host cells to allow them to withstand the rigors of the host environment and establish a suitable replication niche. Changes in host cell properties and behavior include modification of plasma membrane permeability, induction, or inhibition of phagocytosis or apoptosis, depending on the pathogen and the host cell type. These changes result from the action of bacterial proteins that have either membrane interacting activity (pore-forming toxins or PFTs, lipases) or an enzymatic activity (protease, phosphatase, ubiquitin ligase, glucosidase, ADP ribosyltransferase, ...) $[4,16,50]$. These activity-bearing bacterial proteins are either secreted into the extracellular medium and called exotoxins or toxins, or they can be injected into the host cell cytoplasm through specialized bacterial secretion machineries and are then called effectors. In this review we will use the term toxin in its largest sense including both exotoxins and effectors.

Toxins are produced by many bacterial pathogens, whether gram positive or negative, and contribute to, and are sometimes essential for, virulence. Efforts over the last decades have been focused on determining the activity and targets of toxins and how these toxins affect host cell function and behavior. More recently, a novel concern has appeared in the cellular microbiology field, which is to understand whether cells sense the presence or effects of toxins and whether they mount a response, even in the case of nonimmune cells. After a brief introduction on toxins and the mode of delivery of bacterial proteins into the mammalian cytoplasm, we will discuss recent findings that indicate that the effects of toxins can be sensed and that a response is mounted that depends on the nature and amplitude of the toxic insult as well as the type of affected cell. 


\section{The bacterial toolkit}

Bacterial toxins and effectors are generally either enzymes or pore-forming proteins, notable exceptions are the socalled super-antigens, which induce massive unspecific T-cell activation [4]. Note that many pathogenic bacteria will produce more than one toxin and for those harboring a type 3 secretion system (T3SS), always multiple effectors, sometimes near to 20 different ones [72]. While novel enzymatic activities are continuously reported, the list of characterized activities is already extremely long (Table 1), including activities as diverse as ADP-ribosyltransferase such as cholera toxin [39], phosphatidyl inositol-4, 5-phosphatase as Salmonella SopB [30], MAP kinase kinases acetylase as Yersinia YopJ [52], and MAP kinase phosphatase as Shigella OspF [6].

\section{Pore-forming toxins}

In the context of this review, an important class of toxins is that of pore-forming toxins, which compose approximately a third of all characterized bacterial toxins and are produced by both Gram-positive and Gram-negative bacteria [7, 55]. Most PFTs share the following mode of action: they are released by the bacteria in a soluble, generally monomeric, form that freely diffuses towards target cells. Host cell binding occurs by specific interactions with cell surface molecules that can be either lipids, such as for Listeria monocytogenes listeriolysin O (LLO) or Bacillus thuringiensis crystal toxin; proteins, such as for Streptoccus intermedius Intermedilysin or carbohydrates bound to proteins, such as for Aeromonas hydrophila aerolysin [7, 23]. Once bound to its receptor, the toxin undergoes circular polymerization into ring-like structures, the stoichiometry of which varies amongst toxins. The amphipathic nature of these complexes leads to spontaneous insertion into the membrane. Depending on the toxin, the pore diameter varies from 1 up to $50 \mathrm{~nm}[7,55]$. PFTs are generally produced by bacteria that remain extracellular, and thus poreformation occurs at the plasma membrane. Some bacteria that replicate intracellularly, such as L. monocytogenes and Group A Streptococci, however, also produce PFTs, listeriolysin O (LLO), and streptolysin O (SLO), respectively. Although LLO will form pores in the plasma membrane upon secretion by extracellular Listeria, it is absolutely required for the bacterium to breach the phagosomal membrane allowing escape into the cytoplasm [16].

\section{Delivery of bacterial proteins into the host cell cytoplasm}

Except for lipases, the targets of all the bacterial toxins with enzymatic activity mentioned above reside in the host cytoplasm. Thus the first challenge of these molecules is to get across the plasma membrane, an event that is impossible for a soluble protein if not assisted. Evolution has led to two strategies that will be detailed below: (1) the development of a bacterial injection system that brings the enzyme directly from the bacterial cytoplasm to that of the host, (2) the production of a pore-forming protein that escorts the enzyme to the cytoplasm, mediating both host cell recognition and translocation of the enzyme across membranes.

\section{Type III and IV secretion machineries}

Some Gram-negative bacteria inject their toxins into the cytosol of host cells through bacterial transport machines that function as macromolecular syringes [50]. The best characterized is the type III secretion system (T3SS). T3SSs consist of three parts: a cytoplasmic bulb, a region spanning both the inner and the outer bacterial membranes, and a hollow 'needle' protruding from the bacterial surface. The needle is involved in sensing the presence of host cells and triggers the so-called contact mediated secretion, via mechanisms that remain unclear. Transport of effectors is thought to proceed through the central $25-\AA \AA$ channel of the needle. Access to the host cytoplasm, however, also requires perforation of the eukaryotic plasma membrane. This occurs through the secretion of early effectors such as IpaB for Shigella flexneri or SipB for Salmonella that have a pore-forming capacity and puncture the membrane at the tip of the secretion needle, thus creating a continuous conduit from the bacterial cytoplasm to that of the host. This secretion machinery is thought to have evolved from the flagellar export system.

Whereas T3SSs are the most widely spread secretion systems amongst pathogenic bacteria, some Gram-negative bacteria express a somewhat different secretion apparatus termed the Type IV secretion system (T4SSs) [18]. T4SSs were first identified in plant pathogens but have since been identified in Legionella or Brucella, for which they are crucial to establish infection. The interesting additional property of T4SSs is that they have been shown to transport DNA, in addition to proteins, during infection of plants. Injection of bacterial DNA into mammalian cells has not been documented. Please note that type III and IV secretion systems are solely devoted to introducing bacterial proteins in the host cell cytoplasm. Bacterial secretion of exotoxins such as pore-forming toxins relies on other secretion apparatuses such as type I and type II secretion systems.

Needle type injection machineries have not been found in Gram-positive bacteria. It has, however, been shown that Streptococcus pyogenes utilizes a Cytolysin-mediated translocation (CMT) to introduce an effector protein of streptococcal origin into the cytoplasm of a host cell [40]. $S$. pyogenes produces the pore-forming toxin SLO that 


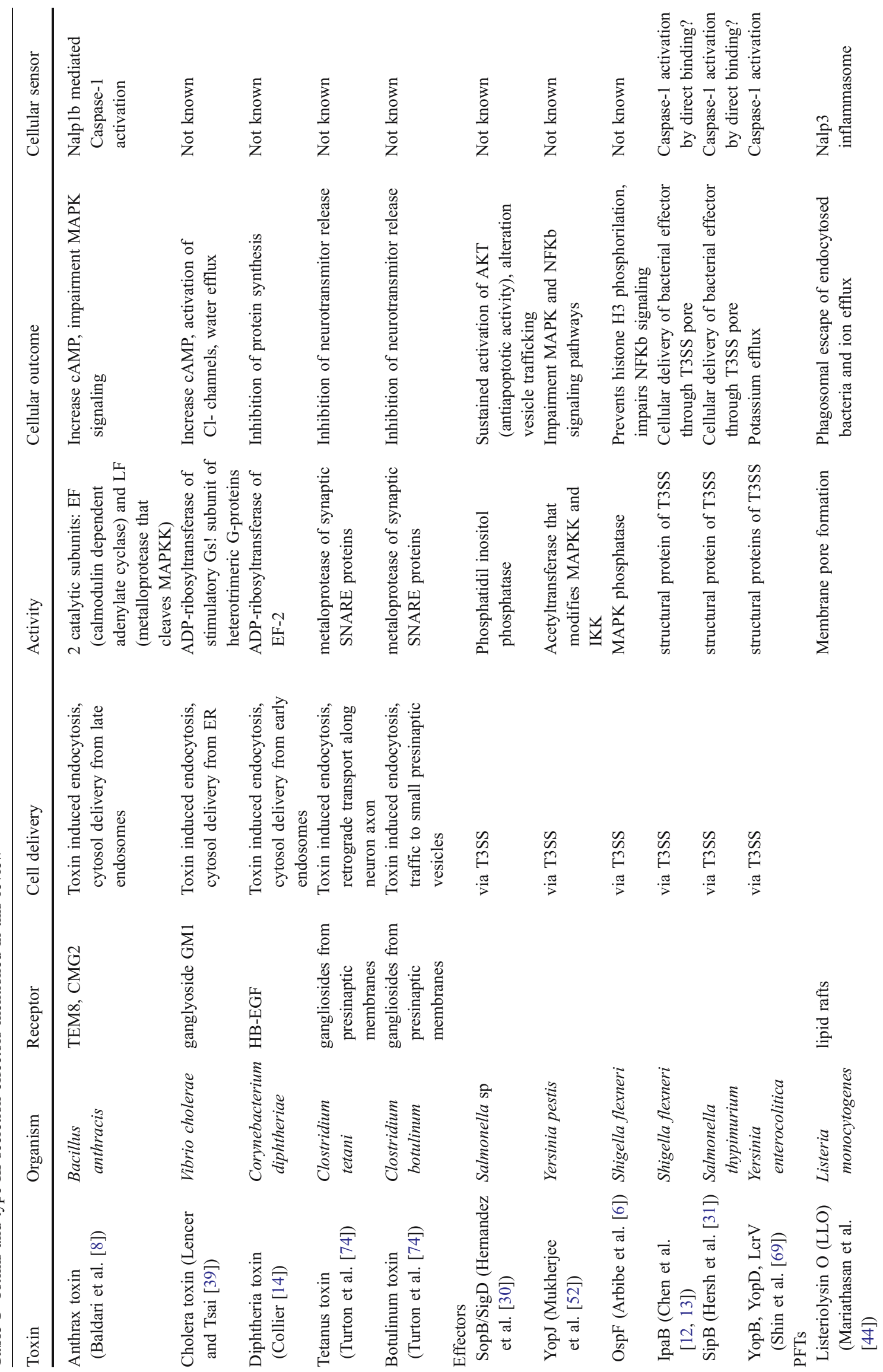




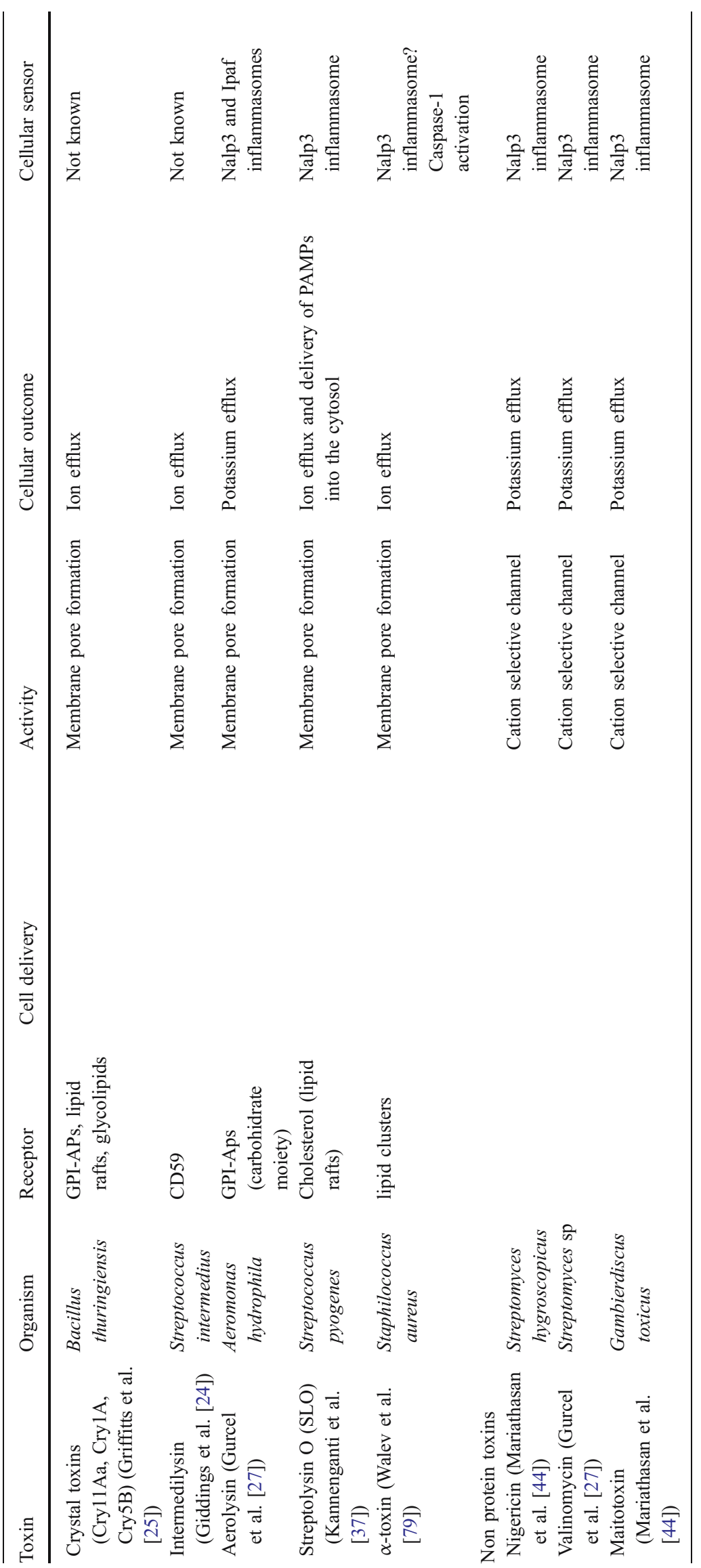


makes large pores which allow the passage of proteins. It was found that the $S$. pyogenes NAD-glycohydrolase involved in virulence is translocated into the host cell via the SLO pore. The model proposes that translocation, which might involve contact between the bacterium and the host, is coupled to polarized secretion, as is supported by the fascinating observation that $S$. pyogenes has a specialized microdomain in its plasma membrane, positioned at a hemispherical position distal to either cell pole, termed the ExPortal, which contains the Sec translocons [63].

\section{AB type toxins}

Toxins of the $\mathrm{AB}$ type are composed either of multiple domains or multiple independent polypeptides. The A subunit bares the enzymatic activity, while the B subunit mediates binding to the target cell membrane, trafficking within the eukaryotic target cell and for many $A B$ toxins, translocation of the A subunit across cellular membranes. Translocation never occurs across the plasma membrane. Therefore the toxin is internalized by the cell, via pathways that are different from one toxin to another, and transported to a final destination, which can be early endosomes, late endosomes, or the endoplasmic reticulum, depending again on the toxin, from which translocation occurs. Whereas translocation at the ER level is thought to be mediated by the cellular translocon, transport at the level of endosomes is mediated by the $\mathrm{B}$ subunit, which has pore-forming activity [2, 61, 64]. Toxins of the $\mathrm{AB}$ family include Cholera toxin, diphtheria toxin, and anthrax toxin (Table 1), for which we will detail the mode of action because it is most relevant to the present review.

Anthrax toxin is composed of three independent polypeptide chains: PA (protective antigen), EF (edema factor), and LF (lethal factor) [2, 68]. EF is a calmodulin-dependent adenylate cyclase thus leading to the increase in c-AMP in intoxicated cells. LF is a metalloprotease that specifically cleaves MAP kinase kinases such as MEK1 or MKK3. Finally, PA corresponds to the B subunit. PA is not only responsible for binding to the cell surface receptors, but it also mediates binding of the enzymatic subunit, EF and LF, transport into the endocytic pathway and translocation into the cytoplasm. To mediate translocation, PA forms a heptameric channel, very similar to that of pore-forming toxins. Channel formation, however, only occurs at acidic $\mathrm{pH}$, thus requiring transport to an endosomal compartment. Due to the ubiquitous nature of the anthrax toxin receptors, Capillary morphogenesis gene 2 (CMG-2) [67] and Tumor endothelial marker 8 (TEM-8) [10], most if not all cells are sensitive to MAP kinase kinase cleavage by anthrax toxin. However, in terms of pathogenesis, the effect on cells of the immune system is most relevant [8]. It was in particular shown that lethal toxin, i.e., the combination of PA and LF, leads to impaired antigen presentation and synthesis of stimulatory molecules by macrophages and dendritic cells, and for certain genetic backgrounds, death, impaired activation of T cells, and inhibition of B-cell proliferation [8].

\section{Sensing bacterial toxins}

For decades, scientists have been studying the effect of bacterial toxins on mammalian cells. The observed effects where either directly linked to the enzymatic activity of the toxin, such as the inhibition of neurotransmitter release in tetanus toxin treated cells due to the toxin-mediated proteolytic cleavage of proteins involved in synaptic vesicle fusion (Table 1), or related to a somewhat unexplained cellular effect, such as activation of the NF- $\mathrm{KB}$ pathway. Recently, a different way of addressing the effects of toxins has emerged, which is to investigate whether cells are able to sense toxins, or their effects, and whether they respond to the toxic insult $[27,29,35,36,60]$.

Some recent studies indicate that cells can sense the effect of certain toxins, as will be detailed below. The field is, however, still in an embryonic state and, therefore, very few examples will be presented. The notion of sensing toxins is of course reminiscent of that of sensing bacterial pathogens, a field of intense research since the discovery a decade ago that Toll-like receptors (TLR) play an important role in innate immunity [38]. The concept that emerged from this work is that through out evolution from flies to mammals, organisms produce microbial sensing proteins that recognize conserved and often structural components of the microorganisms, called pathogen-associated molecular patterns (PAMPs) [3]. Bacterial cell wall components such as peptidoglycan, bacterial flagellin, and nucleic acid structures, unique to bacteria and viruses, are examples of PAMPs. The first class of these pattern-recognition molecules that were identified were the TLRs, which are transmembrane proteins involved in detection of microbes in the extracellular compartment and in endosomes (for review, see [3]). The field of innate immunity has become even more intense since the discovery of a family of cytoplasmic PAMP detectors, which in analogy to the TLRs have been termed NLRs, or Nod-like receptors [46].

NLRs are multidomain proteins that, similar to TLRs, contain a leucine-rich repeat (LRR) domain thought to be involved in the sensing of PAMPs, a nucleotide-binding and self-oligomerization domain termed NACHT domain, and one or more effector binding domains that can be either caspase recruitment domains (CARD) or pyrin effector domains PYD (for review, see [45, 46, 73]). NLRs comprise two large subclasses, the CARD-containing nucleotide oligomerization domain or NODs (NOD1-5) and the PYD-containing domain or Nalps (NACHT-LRR- 
PYD-containing protein) group (Nalp1 to Nalp14). For detailed review on NLRs, their structure, regulation, and ligands, the reader is referred to other reviews in this issue. As for TLRs, NLRs are thought to recognize molecular patterns frequently encountered in microorganisms, such as flagellin or fragments of peptidoglycan. As for TLRs, formal evidence that binding is direct is still missing with the exception of Nalp1, for which direct activation by muramil dipeptide, a fragment of proteoglycan has been reconstituted in vitro with purified molecules [19]. The principle of NLR function is that the molecule is in an autorepressed state, possibly through intramolecular domain interaction, in the absence of stimulus. Upon ligand binding, the molecule opens up rendering the NACHT domain accessible for homo or hetero oligomerization with other NACHT-containing proteins and the CARD and/or PYR domains accessible for the interaction with down stream effectors and/or adaptor proteins. The structural opening of the NLRs upon ligand binding thus induces the assembly of large multiprotein complexes. The two major documented down stream effects of NLR activation are NF$\mathrm{KB}$ activation and caspase- 1 activation, events that involve different NLR members. In this paper, we will only discuss NLRs involved in caspase-1 activation.

The NLR containing multiprotein complexes involved in caspase-1 activation have been termed, by Tschopp and coworkers, the inflammasome [45]. Caspase-1 is indeed a key player in the inflammatory response as this cystein protease is responsible for the processing of the pro-forms of interleukins $1 \beta 18$ and 33, crucial pro-inflammatory cytokines. To date, three different inflammasomes have been described contain- ing Nalp1, Nalp3, and Ipaf (also a NLR family member), respectively (see Fig. 1). Interactions of these NLRs with caspase-1 may be either direct, as proposed for Ipaf (Fig. 1), or require the adaptor protein ASC (Apoptosis-associated Speck-like protein containing a CARD) [46].

As apparent above, both TLRs and NLRs are involved in the detection of common features amongst pathogenic organisms (peptidoglycan, flagellin, ...). As toxins, even within a given family, differ greatly in terms of sequence and even structure, it seems rather unlikely that either TLRs or NLRs would detect the presence of a toxin per se. More likely, if involved, these sensing devices should detect the consequences of the toxins action, which would be similar for toxins with similar activities, enzymatic, or membrane impairing. We will focus on three topics: pore-forming toxins (PFTs) - the largest class of bacterial toxins - anthrax toxin, and finally the type III and IV secretion systems.

\section{Inflammasome activation by pore-forming toxins}

Pore formation leads to permeabilization of the plasma membrane to ions, and in particular to calcium and potassium, along their electrochemical gradients, leading to changes in cytoplasmic ion composition. The large pores formed by certain PFTs as the cholesterol dependent toxins LLO, SLO, and pneumolysin allow also the efflux of cytoplasmic proteins $[32,75]$. As a consequence of pore formation by Staphylococcal $\alpha$ toxin, aerolysin, and LLO, cells of the immune system such as lymphocytes or dendritic cells were found to produce numerous inflamma-
Fig. 1 Schematic diagram of the various inflammasome that have been documented to assemble in response to bacterial toxins or effectors of type III secretion machineries
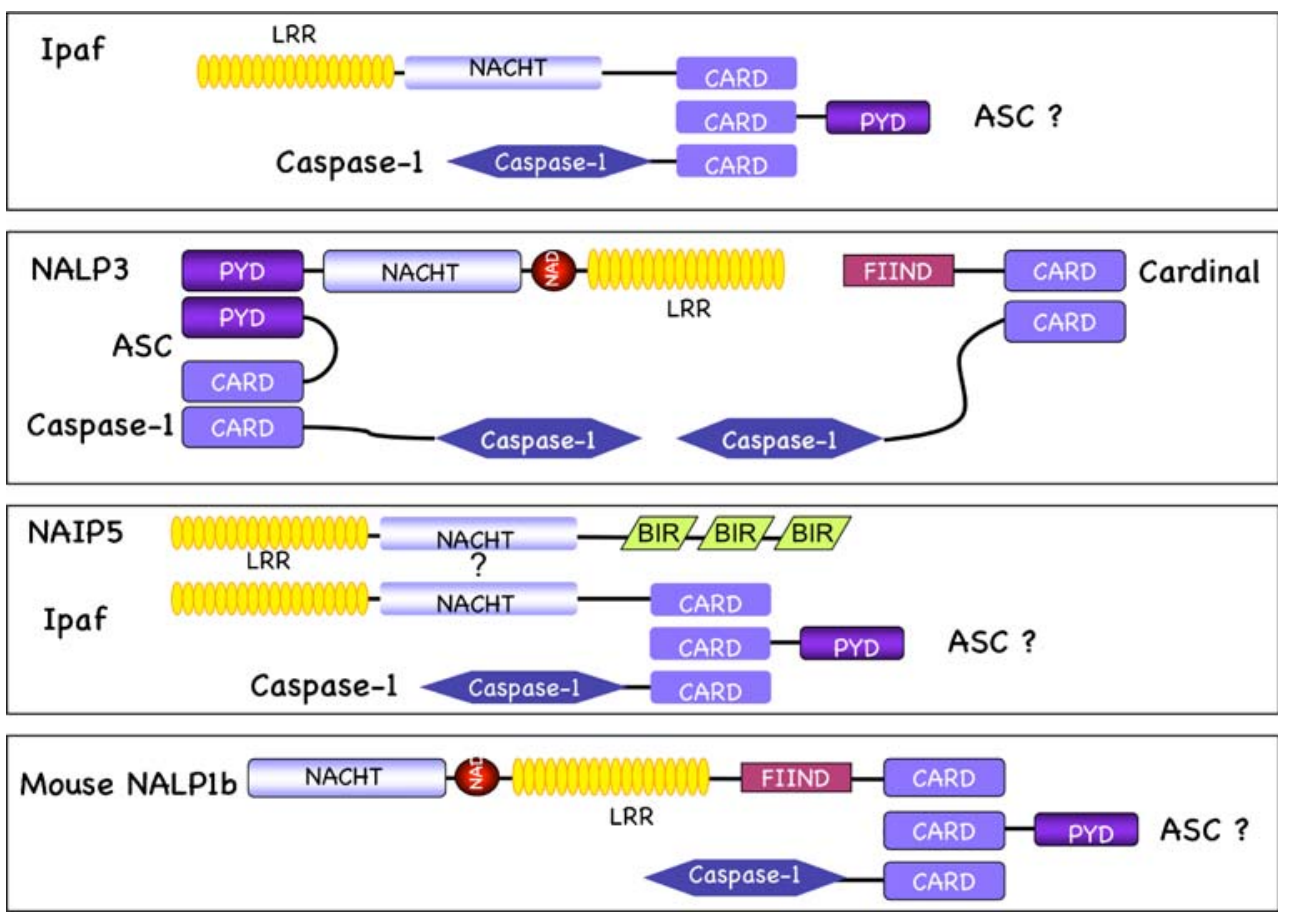
tory molecules and also to undergo apoptosis [7]. The first evidence that PFTs might activate the inflammasome was the observation that alpha-toxin from Staphylococcus aureus induces the release of IL-1 $\beta$ in LPS pretreated monocytes [79]. In these early days, it was already suggested that alpha-toxin triggers the activation of Interleukin-converting enzyme or ICE, the former name of caspase-1. Interestingly, these authors found that high extracellular potassium would prevent toxin induced IL$1 \beta$ secretion, indicating the requirement for a decrease in intracellular potassium.

More recently, it was found that aerolysin from $A$. hydrophila, a toxin that shares the same mode of action as Staphylococcal alpha toxin and makes pores of similar size $[1,28]$, also triggers the activation of caspase-1 [27]. With the increased general knowledge about caspase-1 activation, it was shown that aerolysin induced activation of caspase-1 involves both Nalp3 and Ipaf, in a potassium efflux dependent manner. Caspase-1 activation via the Nalp3 pathway also required the adaptor protein ASC (Fig. 1). Infection of primary human fibroblasts with $A$. hydrophila also triggered caspase-1 activation, in contrast to the isogenic aerolysin deficient strain [27]. In contrast, Nalp3-dependent caspase-1 activation was still observed in $S$. aureus deficient in either alpha, beta, or gamma toxin suggesting that absence of one of the multiple PFTs produced by this bacterium is insufficient to abrogate caspase-1 activation [44]

Thus one of the sensing events that could be common to all PFTs would be the decrease in cytoplasmic potassium (changes in other ions could also be sensed, possibly by other NLRs), which would trigger caspase-1 activation. Potassium ionophores such as nigericin from Streptomyces hygroscopicus [44] and valinomycin [27] have also been found to trigger activation of caspase-1 via Nalp3 inflammasome. In vitro studies have recently shown that NALP inflammasome assembly and caspase-1 recruitment can actually occur spontaneously at $\mathrm{K}^{+}$concentrations below $90 \mathrm{mM}$ [59]

Potassium efflux can also be triggered by the activation of the purinergic $\mathrm{P} 2 \mathrm{X}_{7}$ receptor by ATP, which has long been used as a means to activate caspase-1 [58]. Activation of the receptor-linked cation selective channel triggers a series of events that are not fully understood. First opening of potassium selective channels occurs, followed by the opening of a larger pore, corresponding to the hemichannel pannexin-1 [56]. Surprisingly, pannexin-1 was recently shown to also be crucial for nigericin induced caspase-1 activation as well as that induced by maitotoxin [57]. Maitotoxin is a potent marine nonprotein toxin isolated from the dinoflagellate Gambierdiscus toxicus [80] for which the exact mode of action has not been characterized. This highly cyclic molecule [53] (actually the largest nonprotein molecule known!) first triggers an increase in cytoplasmic calcium, followed by the opening of medium size pores that allow the passage of $\angle 800$ Da molecules, finally followed by the formation of large "death channels." Combined, these studies suggest that the initial change in cytoplasmic ion composition triggered by nigericine or maitotoxin is necessary but not sufficient to trigger caspase-1 activation, which requires pannexin-1, although apparently not through its channel-forming activity [57]. Additional studies will be required to clarify the role of pannexin. It will also be of interest to test whether caspase-1 activation induced by Staphylococcal alpha toxin and aerolysin also involves pannexin-1.

Pannexin-1 channels are reminiscent of the PFTs that form large pores such as SLO and listeriolysin O (LLO), in that they allow the passage of proteins. The similarity was recently extended by the observation that both ATP-induced opening of pannexin-1 channels and SLO allowed the cytosolic access of various PAMPs, such as LPS, synthetic lipid A, lipoteichoic acid, or peptidoglycan, leading to the activation of caspase-1 [37]. LLO was also recently found to be implicated in the caspase-1 activation, via the Nalp3 and ASC inflammasome by L. monocytogenes [44, 54]. $L$. monocytogenes is a Gram-positive facultative intracellular pathogen that can cause life-threatening infections in neonates and immunocompromised hosts. The bacterium enters into cells via a clathrin-mediated pathway [78] and then escapes into the cytoplasm through LLO mediated rupture of the phagosomal membrane [16]. Macrophages cultured with L. monocytogenes deficient in LLO failed to secrete IL-1 $\beta[44,54]$. It is however not clear at what step LLO pore formation was involved in caspase-1 activation: pore formation in the plasma membrane, as recently suggested [71] or in the phagosome? Was cytoplasmic release of the bacterium the required event? In other words, was inflammasome assembly triggered by changes in ion composition or by the presence of PAMPs or both?

\section{Involvement of NLRs in sensing and responding to anthrax toxin}

The second toxin to which cells seem to respond in an inflammasome dependent manner is anthrax toxin. Macrophages treated with anthrax lethal toxin were found to secrete IL-1 $\beta$ and IL-18 $[9,15]$. Direct evidence for the lethal toxin induced activation of caspase-1 was recently obtained by Boyden and Dietrich and confirmed by others ([51], Reig et al., submitted). Searching for an anthrax susceptibility gene in mouse macrophages, Boyden and Dietrich found that Nalp1b was involved in lethal toxin induced cell death in mouse macrophages, in a genetic background dependent manner. These authors found that the gene encoding for Nalp $1 \mathrm{~b}$ is highly polymorphic, with 5 
alleles identified among 18 mice strains. Allele 1, found for example in Balb/cJ, C3H/HeJ, and 129S1/svImJ mice, was found to encode a Nalp1b protein (termed type $1 \mathrm{Nalp} 1 \mathrm{~b}$ ) that was able to mediate caspase-1 activation in response to lethal toxin. In contrast, allele 2, found in C57B6 mice, and allele 4 , found in DBA/2J mice, encodes a Nalp1b protein that is unable to activate caspase-1 in response to LT (termed type $2 \mathrm{Nalp1b}$ ). Whether ASC is required for the type 1 Nalp $1 \mathrm{~b}$ mediated activation of caspase-1 requires further investigation.

The mechanisms that leads to activation of Nalp1b in response to lethal toxin are unclear. It is unlikely, at least for two reasons, that the lethal factor is itself recognized by Nalp1b as a PAMP. As mentioned previously, considering the diversity of bacterial proteins that reach the cytoplasm, it is unlikely that there would be proteins to sense them, the number of sensing proteins would have to be too great. Secondly, the catalytic activity of lethal factor is required to trigger caspase- 1 activation, as a translocation competent but catalytically inactive LF was unable to trigger caspase-1 activation, despite its delivery to the cytoplasm (Reig et al., submitted). Sensing thus appears to occur down stream of MAPK kinase cleavage by LF. Determining the sensed events or component will be of great interest.

\section{Bacterial secretion machineries and the inflammasome}

A variety of Gram-negative bacteria, such as Salmonella and Shigella, that enter and multiply within cells absolutely require their T3SSs for invasion. Upon infection of macrophages, both these bacteria induce robust caspase-1 activation, which leads to the release of IL- $1 \beta$ and IL-18 followed by caspase- 1 mediated death $[12,31,34,65,77]$. The proteins Salmonella SipB and Shigella IpaB, which mediate puncturing of the host cell membrane upon Salmonella and Shigella invasion, respectively, seem to have a direct role in caspase-1 induced cell death as microinjection of recombinant $\mathrm{SipB}$ or IpaB protein is sufficient to induce apoptosis in macrophages [13, 31]. Furthermore, Salmonella and Shigella strains defective in IpaB or SipB do not induce macrophage apoptosis [31, 34]. Although direct binding of IpaB and SipB was thought to activate caspase-1 [31,34], more recent data suggest that $\mathrm{SipB}$ has a more indirect role in caspase-1 activation during infection with Salmonella sp. [22, 47]. Since in the absence of these proteins, none of the other effectors can reach the cytosol, it is unclear whether inflammasome activation is triggered by the secretion system, or some of the effectors, or PAMPs that gain access through IpaB or $\mathrm{SipB}$ channels. It was recently shown that the hydrophobic translocators of Yersinia, YopB and YopD, induce caspase-1 activation and IL- $1 \beta$ secretion upon infection with strains that are deficient in effectors [69].
These observations highlight the importance of membrane impairment in triggering inflammation. Interestingly upon infection with a Yersinia wild type strain, caspase-1 activation did not occur, suggesting that the effectors neutralize the proinflammatory phenomenon of the translocator either by inhibiting caspase- 1 activation through the GTPase activity of the effector protein YopE (Schotte, Denecker et al. 2004) or possibly by clogging the translocon [69].

Infection by $S$. typhymurium and S. flexneri, however, appears to trigger the activation of more than one type of inflammasome as both ASC and Ipaf were found to be involved in activating caspase-1 [43]. Only the Ipaf inflammasome was, however, essential for caspase-1 dependent cell death [42]. Although still requiring SipB, S. typhimurium flagellin was found to trigger Ipaf mediated caspase-1 activation as flagellin deficient Salmonella did not activate caspase-1 [22, 47, 62]. Furthermore, Ipaf activation could be recapitulated by the introduction of purified flagellin directly in the cytoplasm. Altogether, these studies indicate that bacteria may trigger caspase-1 activation via more than one pathway.

Activation of caspase-1 has also been observed for other Gram-negative such as the facultative intracellular pathogen Burkholderia pseudomallei, which harbors a T3SS highly homologous to that of $S$. typhymurium and S. flexneri. B. pseudomallei is the causative agent for meliodosis, an infection that results in a wide spectrum of clinical outcomes including asymptomatic, acute or chronic pneumonia, or septicemia [11]. B. pseudomallei infected macrophages where found to undergo caspase-1 dependent cell death. The inflammasome components mediating cell death were however not determined, nor was the role of BipB, the Burkholderia homologue of IpaB.

Finally, Legionella pneumophila, a T4SS harboring Gram-negative intracellular pathogen was also found to trigger caspase-1 activation [82]. Activation was strictly dependent of Dot-Icm type IV secretion system and led to the assembly of two NLR containing complexes: a Bircle (Naip5) and an Ipaf inflammasome that were sensitive to bacterial flagellin $[5,62]$.

\section{Interplay between TLRs and inflammasomes}

Activated caspase- 1 is essential for the processing and release of biologically active IL-1 $\beta$ and IL-18, two proinflammatory cytokines with critical roles in innate and adaptive immunity [17]. The secretion of IL-1 $\beta$ is also dependent on the levels of pro-IL- $1 \beta$ induced at the transcriptional level by proinflammatory stimuli including several TLR ligands such as lipopolysaccharide (LPS), lipoteichoic acid (LTA), CpG oligonucleotides, the antiviral compound resiquimod R848, and the lipopeptide Pam3CSK4 [41]. The crucial signal 
transduced by TLRs for the induction of pro-IL- $1 \beta$ expression is likely mediated by the transcription factor NF- $\mathrm{kB}$. The cytokine IL-18 is also synthesized as an inactive precursor, but, unlike pro-IL-1 $\beta$, it is expressed constitutively. Despite the importance of TLR activation in pro-IL-1 $\beta$ synthesis, the relationship between TRL and inflammasome signaling remains poorly understood.

Several lines of evidence indicate that caspase-1 activation does not depend on TLR signaling. It has indeed been shown that inhibition of TLR signaling, for example, upon knock out of MyD88 or Toll/IL-1 receptor (TIR)-domain-containing adaptor-inducing IFN- $\beta$ (TRIF), two adaptor molecules that are critical for TLR mediated NF- $\mathrm{KB}$ activation, up-regulation of pro-IL-1 $\beta$ does not occur but processing of caspase-1 is unaltered [37, 54, 81]. In contrast, recent data now indicate that TLR signaling can be regulated by caspase-1. Upon ligand activation of TLRs, cytosolic TIR domain-containing adapter proteins are recruited to initiate signaling events [48]. Among them, the MyD88 adaptor-like (MAL) protein is required for signaling by the LPS receptor TLR4 and the bacterial lipoprotein receptor TLR2 and acts as a bridging adapter for MyD88 recruitment [48]. Recent results reveal that MAL requires cleavage by caspase-1 to elicit its downstream signal NF-kB [49]. Consequently, signaling by TLR2 and TLR4 is impaired in caspase-1 deficient cells. NFKB activation via Receptor Interacting Protein-2 (RIP2) was also found to involve caspase-1 [66]. In this case also, NF-KB activation by LPS was attenuated in caspase- 1 deficient macrophages and was inhibited by a catalytically inactive form of caspase-1. Furthermore, the authors point out a possible regulatory role of ASC in the communication between the inflammasome and the NF- $k B$ pathway. Overexpression of ASC led to a decrease in NF- $\mathrm{kB}$ activity, whereas ASC knock down led to an increase in NF- $\mathrm{KB}$ activity suggesting an inhibitory role of ASC in NF- $\mathrm{KB}$ signaling. These findings shed light on an important mechanism for the coordinated activation of host defense responses by TLRs and the inflammasome.

At present, there is no evidence that toxins trigger TLRs. Il-1 $\beta$ secretion upon exposure to pore-forming toxins or anthrax toxin might be due to the synergistic effect of bacterial PAMPs and the toxins. Cellular damage due to bacterial toxins, in particular, PFTs, could also lead to the release of endogenous TLR ligands such as heat shock proteins [76] or other danger signals.

\section{Consequences of toxins induced caspase- 1 activation}

The consequences of toxin-induced caspase- 1 activation appear to be strongly cell type dependent. In cells of the immune system such as macrophages, the most striking consequence of caspase-1 activation by either the poreforming effectors of T3SS harboring bacteria or the totally unrelated anthrax toxin is cell death, in addition to processing and secretion of IL-1 $\beta$ and IL-18 $[9,13,31,33]$. The underlying mechanisms, however, remain completely obscure and probably involve yet to be identified targets of caspase-1 (Fig. 2). Caspases-2, 3, and 11 do not seem to be involved in this process, and $\mathrm{Bcl}-2$ overexpression does not block
Fig. 2 Schematic representation of bacterial toxins and effectors that activate NLRs, trigger the assembly of inflammasomes and the activation of caspase-1

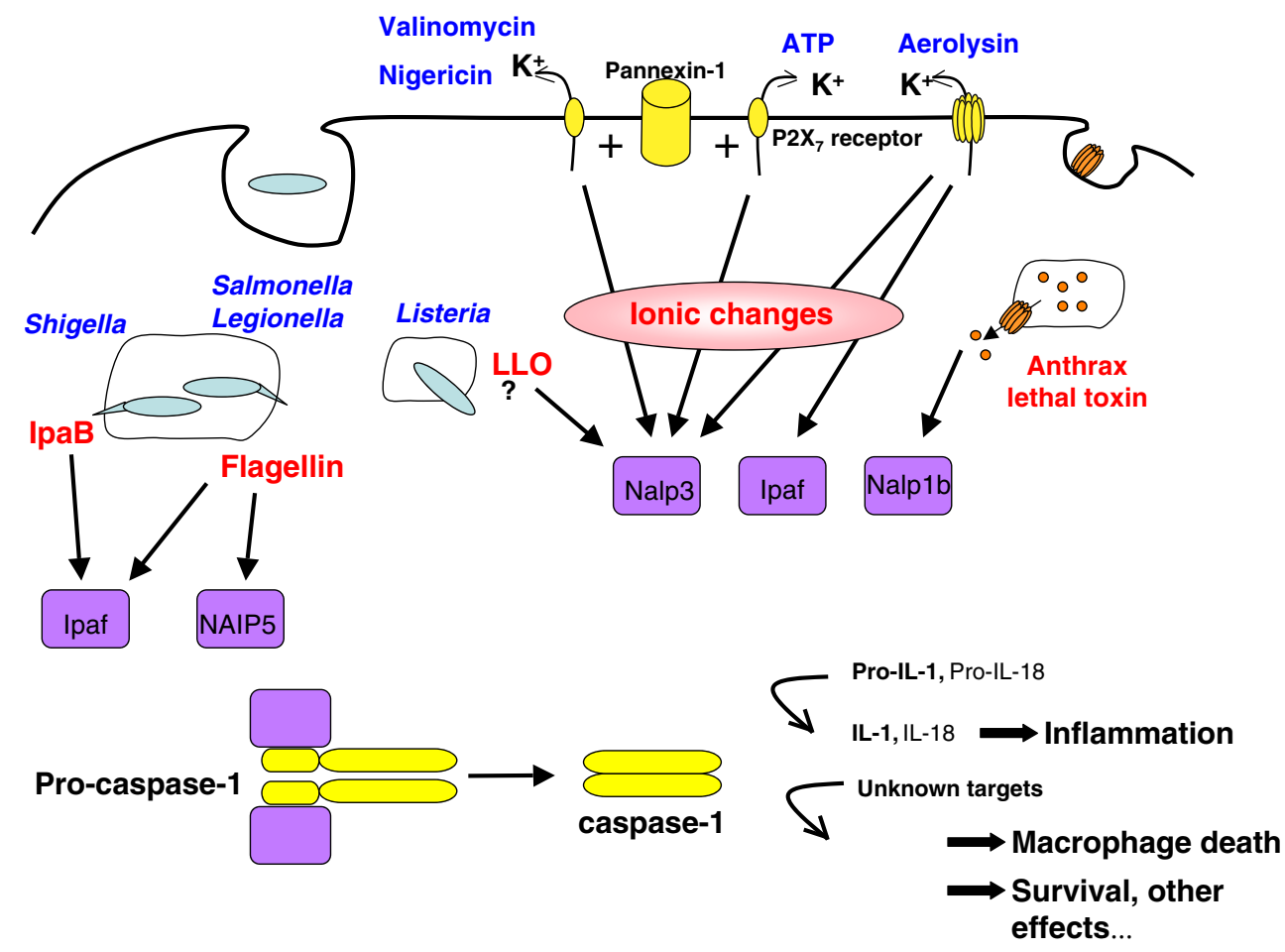


Shigella-induced cell death in macrophages [34]. Several lines of evidence suggest that caspase-1 induced cell death is mechanistically distinct from the forms of classical apoptosis and was therefore termed pyroptosis [20]. An interesting event described in pyroptosis is the caspase-1 dependant formation of plasma membrane pores, between 1.1 and $2.4 \mathrm{~nm}$ in diameter, that appear concurrently to the release of processed IL-1 $\beta$ and IL-18 and precede cell death [21]. These pores also dissipate ionic gradients leading to water uptake and cell swelling, ultimately culminating in osmotic lysis with release of inflammatory cellular contents. Despite of their difference in size, these membrane pores are reminiscent of the pannexin-1 pore mentioned above [56].

An interesting aspect of caspase-1 mediated cell death in response to anthrax lethal toxin, and presumably others to come, is that cell death is not a direct consequence of the toxins action, as illustrated by the fact that nonimmune cells survive toxin action for days (unpublished observations). Rather, death of immune cells is due to the innate response of the cells to the action of the toxin. This theme is reminiscent of what has been observed in infection where tissue damage is often the results of a massive immune response rather that the action of the bacterium itself.

Many toxins however target nonimmune cells such as epithelial cells. This is in particular the case for the poreforming toxin aerolysin produced by the enteropathogen $A$. hydrophila. Aerolysin was shown to trigger caspase-1 activation both in fibroblast like cells [27] as well as human intestinal cells (Freche and van der Goot, unpublished). These cells express low to undetectable level of Il-1 $\beta$ and thus caspase-1 does not appear to mediate cytokine secretion. These cells are however sensitive to caspase-1 activation. We have recently shown that caspase-1 triggers the activation of the central regulators of lipid metabolism, the sterol responsive element binding proteins (SREBPs) after treatment of epithelial cells with aerolysin and Staphylococcal $\alpha$ toxin [27]. SREBPs are membrane-bound transcription factors that control the synthesis of enzymes involved in lipid metabolism [26]. Caspase-1 mediated SREBP activation was found to promote cell survival after toxin attack. Although SREBP activation occurs via a proteolytic mechanism, cleavage required the traditional site-1 and site 2-protease indicating that caspase-1 was processing a yet to be identified substrate involved up stream of SREBP.

\section{Concluding remarks}

The exquisite action of bacterial toxins on cellular functions of the target cells is a consequence of a long tem coevolution between bacteria and host. But evolution has not only shaped the pathogen but clearly also the host and recent reemergence of the innate immunity field has provided beautiful examples of how hosts detect the presence of pathogens through the present of particular patterns. Toxins however appear to be detected through their effects rather than by their structure, which are extremely diverse and show not conserved patterns. The coming years are likely to be exciting as sensors for the effect of toxins will be identified and understood.

\section{References}

1. Abrami L, Fivaz M et al (2000) Adventures of a pore-forming toxin at the target cell surface. Trends Microbiol 8(4):168-172

2. Abrami L, Reig N et al (2005) Anthrax toxin: the long and winding road that leads to the kill. Trends Microbiol 13(2):72-78

3. Akira S, Uematsu S et al (2006) Pathogen recognition and innate immunity. Cell 124(4):783-801

4. Alouf JE, Freer JH (eds) (2005) The comprehensive sourcebook of bacterial protein toxins. Academic Press, London

5. Amer A, Franchi L et al (2006) Regulation of Legionella phagosome maturation and infection through flagellin and host Ipaf. J Biol Chem 281(46):35217-35223

6. Arbibe L, Kim DW et al (2007) An injected bacterial effector targets chromatin access for transcription factor NF-kappaB to alter transcription of host genes involved in immune responses. Nat Immunol 8(1):47-56

7. Aroian R, van der Goot FG (2007) Pore-forming toxins and cellular non-immune defenses (CNIDs). Curr Opin Microbiol 10(1):57-61

8. Baldari CT, Tonello F et al (2006) Anthrax toxins: a paradigm of bacterial immune suppression. Trends Immunol 27(9):434-440

9. Boyden ED, WF Dietrich (2006) Nalp1b controls mouse macrophage susceptibility to anthrax lethal toxin. Nat Genet 38(2):240-244

10. Bradley KA, Mogridge $J$ et al (2001) Identification of the cellular receptor for anthrax toxin. Nature 414(6860):225-229

11. Chaowagul W, White NJ et al (1989) Melioidosis: a major cause of community-acquired septicemia in northeastern Thailand. J Infect Dis 159(5):890-899

12. Chen LM, Kaniga K et al (1996) Salmonella spp. are cytotoxic for cultured macrophages. Mol Microbiol 21(5):1101-1115

13. Chen Y, Smith MR et al (1996) A bacterial invasin induces macrophage apoptosis by binding directly to ICE. Embo J 15 (15):3853-3860

14. Collier RJ (1990) Diphtheria toxin: Structure and function of a cytocidal protein. ADP-ribosylating toxins and G proteins: Insights into signal transduction. J. M. a. M. Vaughan. Washington DC, American Society of Microbiology: 3-19

15. Cordoba-Rodriguez R, Fang $\mathrm{H}$ et al (2004) Anthrax lethal toxin rapidly activates caspase-1/ICE and induces extracellular release of interleukin (IL)-1beta and IL-18. J Biol Chem 279(20):20563-20566

16. Cossart P, Sansonetti PJ (2004) Bacterial invasion: the paradigms of enteroinvasive pathogens. Science 304(5668):242-248

17. Dinarello CA (1998) Interleukin-1 beta, interleukin-18, and the interleukin-1 beta converting enzyme. Ann N Y Acad Sci 856:1-11

18. Ding Z, Atmakuri K et al (2003) The outs and ins of bacterial type IV secretion substrates. Trends Microbiol 11(11):527-535

19. Faustin B, Lartigue L et al (2007) Reconstituted Nalp1 inflammasome reveals two-step mechanism of caspase-1 activation. Mol Cell 25:713-724

20. Fink SL, Cookson BT (2005) Apoptosis, pyroptosis, and necrosis: mechanistic description of dead and dying eukaryotic cells. Infect Immun 73(4):1907-1916 
21. Fink SL, Cookson BT (2006) Caspase-1-dependent pore formation during pyroptosis leads to osmotic lysis of infected host macrophages. Cell Microbiol 8(11):1812-1825

22. Franchi L, Amer A et al (2006) Cytosolic flagellin requires Ipaf for activation of caspase-1 and interleukin 1beta in salmonellainfected macrophages. Nat Immunol 7(6):576-582

23. Geny B, Popoff MR (2006) Bacterial protein toxins and lipids: Pore formation or toxin entry into cells. Biol Cell 98(11):667678

24. Giddings KS, Zhao J et al (2004) Human CD59 is a receptor for the cholesterol dependent cytolysin intermedilysin. Nat Struct Mol Biol 11:1173-1178

25. Griffitts JS, Haslam SM et al (2005) Glycolipids as receptors for Bacillus thuringiensis crystal toxin. Science 307:922-925

26. Goldstein JL, DeBose-Boyd RA et al (2006) Protein sensors for membrane sterols. Cell 124(1):35-46

27. Gurcel L, Abrami L et al (2006) Caspase-1 dependent activation of SREBPS promotes cell survival in response to bacterial poreforming toxins. Cell 126:1135-1145

28. Gurcel L, Iacovache I et al (2005) Aerolysin and related Aeromonas toxins. The comprehensive sourcebook of bacterial protein toxins. JE Alouf, JH Freer, Academic, London

29. Haugwitz U, Bobkiewicz W et al (2006) Pore-forming Staphylococcus aureusalpha-toxin triggers epidermal growth factor receptordependent proliferation. Cell Microbiol 8(10):1591-1600

30. Hernandez LD, Hueffer K et al (2004) Salmonella modulates vesicular traffic by altering phosphoinositide metabolism. Science 304(5678):1805-1807

31. Hersh D, Monack DM et al (1999) The Salmonella invasin SipB induces macrophage apoptosis by binding to caspase-1. Proc Natl Acad Sci USA 96(5):2396-2401

32. Heuck AP, Tweten RK et al (2001) Beta-barrel pore-forming toxins: intriguing dimorphic proteins. Biochemistry 40(31):90659073

33. Hilbi H, Chen Y et al (1997) The interleukin 1beta-converting enzyme, caspase 1, is activated during Shigella flexneri-induced apoptosis in human monocyte-derived macrophages. Infect Immun 65(12):5165-5170

34. Hilbi H, Moss JE et al (1998) Shigella-induced apoptosis is dependent on caspase-1 which binds to IpaB. J Biol Chem 273 (49):32895-32900

35. Huffman DL, Abrami L et al (2004) Mitogen-activated protein kinase pathways defend against bacterial pore-forming toxins. Proc Natl Acad Sci USA 101(30):10995-11000

36. Husmann M, Dersch K et al (2006) Differential role of p38 mitogen activated protein kinase for cellular recovery from attack by pore-forming S. aureus alpha-toxin or streptolysin O. Biochem Biophys Res Commun 344(4):1128-1134

37. Kanneganti TD, Lamkanfi $M$ et al (2007) Pannexin-1-mediated recognition of bacterial molecules activates the Cryopyrin inflammasome independent of Toll-like receptor signaling. Immunity 26 (4):433-443

38. Lemaitre B, Nicolas E et al (1996) The dorsoventral regulatory gene cassette spatzle/Toll/cactus controls the potent antifungal response in Drosophila adults. Cell 86(6):973-983

39. Lencer WI, Tsai B (2003) The intracellular voyage of cholera toxin: going retro. Trends Biochem Sci 28(12):639-645

40. Madden JC, Ruiz N et al (2001) Cytolysin-mediated translocation (CMT): A functional equivalent of type III secretion in grampositive bacteria. Cell 104(1):143-152

41. Mariathasan S (2007) ASC, Ipaf and Cryopyrin/Nalp3: Bona fide intracellular adapters of the caspase-1 inflammasome. Microbes Infect 9(5):664-671

42. Mariathasan S, Monack DM (2007) Inflammasome adaptors and sensors: intracellular regulators of infection and inflammation. Nat Rev Immunol 7(1):31-40
43. Mariathasan S, Newton K et al (2004) Differential activation of the inflammasome by caspase-1 adaptors ASC and Ipaf. Nature 430(6996):213-218

44. Mariathasan S, Weiss DS et al (2006) Cryopyrin activates the inflammasome in response to toxins and ATP. Nature 440 (7081):228-232

45. Martinon F, Burns K et al (2002) The inflammasome: a molecular platform triggering activation of inflammatory caspases and processing of proIL-beta. Mol Cell 10(2):417-426

46. Martinon F, Tschopp J (2005) NLRs join TLRs as innate sensors of pathogens. Trends Immunol 26(8):447-454

47. Miao EA, Alpuche-Aranda CM et al (2006) Cytoplasmic flagellin activates caspase-1 and secretion of interleukin 1beta via Ipaf. Nat Immunol 7(6):569-575

48. Miggin SM, O’Neill LA (2006) New insights into the regulation of TLR signaling. J Leukoc Biol 80(2):220-226

49. Miggin SM, Palsson-McDermott E et al (2007) NF-kappaB activation by the Toll-IL-1 receptor domain protein MyD88 adapter-like is regulated by caspase-1. Proc Natl Acad Sci USA 104(9):3372-3377

50. Mota LJ, Sorg I et al (2005) Type III secretion: The bacteriaeukaryotic cell express. FEMS Microbiol Lett 252(1):1-10

51. Muehlbauer SM, Evering TH et al (2007) Anthrax lethal toxin kills macrophages in a strain-specific manner by apoptosis or caspase-1-mediated necrosis. Cell Cycle 6(6):758-766

52. Mukherjee S, Keitany G et al (2006) Yersinia YopJ acetylates and inhibits kinase activation by blocking phosphorylation. Science 312(5777):1211-1214

53. Nicolaou KC, Frederick MO (2007) On the Structure of Maitotoxin. Angew Chem Int Ed Engl

54. Ozoren N, Masumoto J et al (2006) Distinct roles of TLR2 and the adaptor ASC in IL-1beta/IL-18 secretion in response to Listeria monocytogenes. J Immunol 176(7):4337-4342

55. Parker MW, Feil SC (2005) Pore-forming protein toxins: From structure to function. Prog Biophys Mol Biol 88(1):91-142

56. Pelegrin P, Surprenant A (2006) Pannexin-1 mediates large pore formation and interleukin-1beta release by the ATP-gated P2X7 receptor. Embo J 25(21):5071-5082

57. Pelegrin P, Surprenant A (2007) Pannexin-1 couples to maitotoxin- and nigericin-induced interleukin-1beta release through a dye uptake-independent pathway. J Biol Chem 282 (4):2386-2394

58. Perregaux D, Gabel CA (1994) Interleukin-1 beta maturation and release in response to ATP and nigericin. Evidence that potassium depletion mediated by these agents is a necessary and common feature of their activity. J Biol Chem 269 (21):15195-15203

59. Petrilli V, Papin S, Dostert C, Mayor A, Martinon F, Tschopp J (2007) Activation of the NALP3 inflammasome is triggered by low intracellular potassium concentration. Cell Death Differ (in press)

60. Ratner AJ, Hippe KR et al (2006) Epithelial cells are sensitive detectors of bacterial pore-forming toxins. J Biol Chem

61. Reig N, van der Goot FG (2006) About lipids and toxins. FEBS Lett 580(23):5572-5579

62. Ren T, Zamboni DS et al (2006) Flagellin-deficient Legionella mutants evade caspase-1- and Naip5-mediated macrophage immunity. PLoS Pathog 2(3):e18

63. Rosch J, Caparon M (2004) A microdomain for protein secretion in Gram-positive bacteria. Science 304(5676):1513-1515

64. Sandvig K, van Deurs B (2002) Transport of protein toxins into cells: Pathways used by ricin, cholera toxin and Shiga toxin. FEBS Lett 529(1):49-53

65. Sansonetti PJ, Phalipon A et al (2000) Caspase-1 activation of IL1beta and IL-18 are essential for Shigella flexneri-induced inflammation. Immunity 12(5):581-590 
66. Sarkar A, Duncan M et al (2006) ASC directs NF-kappaB activation by regulating receptor interacting protein-2 (RIP2) caspase-1 interactions. J Immunol 176(8):4979-4986

67. Scobie HM, Rainey GJ et al (2003) Human capillary morphogenesis protein 2 functions as an anthrax toxin receptor. Proc Natl Acad Sci USA 100(9):5170-5174

68. Scobie HM, Young JA (2005) Interactions between anthrax toxin receptors and protective antigen. Curr Opin Microbiol 8(1):106-112

69. Shin H, Cornelis GR (2007) Type III secretion translocation pores of Yersinia enterocolitica trigger maturation and release of Proinflammatory IL-1beta. Cell Micro (in press)

70. Sun GW, Lu J et al (2005) Caspase-1 dependent macrophage death induced by Burkholderia pseudomallei. Cell Microbiol 7 (10):1447-1458

71. Sutterwala FS, Ogura Y, Flavell RA (2007) The inflammasome in pathogen recognition and inflammation. J Leukoc Biol (in press)

72. Troisfontaines P, Cornelis GR (2005) Type III secretion: more systems than you think. Physiology (Bethesda) 20:326-339

73. Tschopp J, Martinon F et al (2003) NALPs: a novel protein family involved in inflammation. Nat Rev Mol Cell Biol 4(2):95-104

74. Turton K, Chaddock JA et al (2002) Botulinum and tetanus neurotoxins: Structure, function and therapeutic utility. Trends Biochem Sci 27(11):552-558
75. Tweten RK, Parker MW et al (2001) The cholesterol-dependent cytolysins. Curr Top Microbiol Immunol 257:15-33

76. Vabulas RM, Wagner H, Schild H (2002) Heat shock proteins as ligands of toll-like receptors. Curr Top Microbiol Immunol 270:169-184

77. van der Velden AW, Velasquez M et al (2003) Salmonella rapidly kill dendritic cells via a caspase-1-dependent mechanism. J Immunol 171(12):6742-6749

78. Veiga E, Cossart P (2005) Listeria hijacks the clathrin-dependent endocytic machinery to invade mammalian cells. Nat Cell Biol 7 (9):894-900

79. Walev I, Reske K et al (1995) Potassium-inhibited processing of IL-1 beta in human monocytes. Embo J 14(8):1607-1614

80. Wisnoskey BJ, Estacion M et al (2004) Maitotoxin-induced cell death cascade in bovine aortic endothelial cells: divalent cation specificity and selectivity. Am J Physiol Cell Physiol 287(2):C345-C356

81. Yamamoto M, Yaginuma K et al (2004) ASC is essential for LPSinduced activation of procaspase-1 independently of TLR-associated signal adaptor molecules. Genes Cells 9(11):1055-1067

82. Zamboni DS, Kobayashi KS et al (2006) The Bircle cytosolic pattern-recognition receptor contributes to the detection and control of Legionella pneumophila infection. Nat Immunol 7 (3):318-325 